\title{
Burns in pregnancy: Five-year experience in a tertiary hospital in southeastern Nigeria
}

\author{
Ugochukwu U. Nnadozie ${ }^{1,2 *}$, Charles C Maduba ${ }^{2}$, Gabriel M. Okorie ${ }^{2}$, Lucky O. Lawani ${ }^{3}$, Anikwe \\ C Chidebe ${ }^{3}$, Obiora G. K Asiegbu ${ }^{3}$, Amaechi Ugbala ${ }^{2}$
}

1. Division of Plastic Surgery, Alex Ekwueme Federal University Teaching Hospital, Nigeria.

2. Division of Plastic Surgery, Department of Surgery

3. Department of Obstetrics and Gynaecology, Alex Ekwueme University Teaching Hospital Abakaliki

Correspondence: Ugochukwu Uzodimma Nnadozie.(ugodozie@yahoo.ca)

\section{Background}

Burns in pregnancy is often associated with high maternal and fetal morbidity and mortality especially when the total burn surface area (TBSA) involved is high. This study aims to review management outcome of cases of burns in pregnancy at Alex Ekwueme Federal University Teaching Hospital Abakaliki (AE-FUTHA).

\section{Methods}

A five year retrospective study of all pregnant women that presented at AE-FUTHA with burn injury between April 2014 and March 2019. Information was collected from the medical records using a proforma and analyzed with IBM SPSS Statistics version 20.0 (IBM Corp., Armonk, NY, USA) using descriptive statistics.

Results

A total of 222 cases of burns were managed but only 8 were pregnant, giving an incidence of 3.6\%. The commonest causes were flame $(62.5 \%)$, scald $(25 \%)$ and friction $(12.5 \%)$ occurring mostly during the harmattan season. The median age of participants was $25-34$ years. The burns affected $12.5 \%$ of the patients in the first trimester and $62.5 \%$ and $25 \%$ in the 2 nd and 3 rd trimesters respectively. Most patients $(62.5 \%)$ had superficial burns while $25 \%$ had other associated injuries in addition to burns. About $87.5 \%$ had term spontaneous vaginal delivery. There was no maternal death but, there was an early neonatal death.

Conclusion

The good outcome observed in this study with a $100 \%$ survival, could be explained by inter-disciplinary management approach given, even as most cases were minor degrees of burns. Early involvement of obstetricians in all burns affecting pregnant women is advised especially in burn centres where obstetricians are hardly in the employ.

Keywords: Burns in pregnancy; maternal outcome; multi-disciplinary management; Nigeria;

\section{Introduction}

Burn injuries in pregnancy are often times life threatening to the mother and her unborn child with increased risk of perinatal and maternal mortality and morbidity, especially if not properly managed. These injuries often result from hot liquids (scald), hot solids (contact burns), or flames (flame burns) which may cause superficial or deep skin damages ${ }^{1}$. Burns can also occur from ultraviolet radiations, radioactive agents, electrical, and chemical sources. Smoke inhalation from flames can result in respiratory tract injuries, especially in cases of fire or toxic chemical exposure ${ }^{1,2}$. Apart from the physical injuries, burns cause emotional, psychological and post-traumatic stress disorder to its victims and constitute a source of financial burden to the family, especially in low resource settings like Nigeria, where the health insurance scheme is not fully operational or still at the teething stage, resulting in out of pocket payment for health care services ${ }^{2,3}$. Burns is a rare occurrence during pregnancy but maybe associated with high complication and deaths rates, especially in the low and middle-income countries (LMIC) where more than $4 / 5$ th of life threatening burns occur ${ }^{4,5}$.

Unfortunately, it is difficult to ascertain the true incidence, morbidity and mortality rates of burns in pregnancy $y^{6,7}$. However, Mabogunje in Zaria, Nigeria reported that $86 \%$ of the parturient who suffer burn injuries and $71 \%$ of their fetuses survived ${ }^{8}$. In order to determine the true incidence of burns in pregnancy, it is thus necessary for all burnt cases of childbearing age women to have a pregnancy test conducted $^{7-9}$.

Maternal and perinatal survival correlate significantly with the degree of burn or Total Burn Surface Area (TBBSA) involved. Furthermore, there are reports that adverse prognosis are commoner in parturient with TBSA between $40-60 \% 0^{4,5}$.

Burns often result in physiological derangement of the normal body functions and processes, as well as result in additional stress on body systems that are already modified, such as the cardiovascular system which becomes hyper dynamic during pregnancy. Following incidents of burns, there are increased capillary permeability and third space losses leading to hypovolemia and hypotension. These may lead to placental insufficiency with fetal compromise or demise and premature delivery if no appropriate and timely interventions are offered ${ }^{11,10}$. Therefore, adequate resuscitation with intravenous fluids and oxygen supplementation are required as part of supportive management ${ }^{11}$.

In early pregnancy, fetal outcome to a large extent depends on gestational age, degree of injury and maternal survival ${ }^{12}$. 
However, beyond 32 weeks, neonatal outcome significantly depends on survival of the woman ${ }^{12}$. Fetal survival sometimes depends on the absence of morbidities such as infection, hemodynamic instability like hypotension and hypoxia ${ }^{7}$. Multi-disciplinary approach between the surgical, obstetric and other teams providing supportive care is necessary to achieve optimal outcome. Premature contractions and labor may be aborted by the use of tocolytics, while the patient is being stabilized'. The timing and mode of delivery are mainly based on obstetric indications. Nevertheless, vaginal delivery is usually preferred in the absence of contra-indications ${ }^{6}$.

Antibiotic that are safe in pregnancy are used. Their use in burn are for prophylaxis, presumptive use and, for treatment of infection ${ }^{13,14}$. Management of wound with tangential excision plus split-thickness skin graft aid wound healing and reduce risk of infection and enhances feto-maternal outcome? .

There are limited publications and current evidence regarding burns in pregnancy, despite being necessary to formulate guidelines for burn-care providers, counseling patients and their families of potential risk, complications and prognosis. This study attempts to provide an update and recent evidence regarding burns in pregnancy, by determining the clinical presentation, management and treatment outcomes of cases managed at the burns and plastic unit of AE-FUTHA.

\section{Methods}

\section{Study design}

This is a retrospective study of pregnant women with burns, managed at the Burns and Plastic Surgery unit of AE-FUTHA over 5 years (April, 2014 to March, 2019). Ethical approval was gotten from the Research and Ethics Committee of AEFUTHA with Approval reference; Ref. no: FETHA/REC/ VOL2/2019/261. Routinely our institution being a teaching hospital usually takes consent from patients they manage on using their information for possible teaching, research and educational purposes of public interest.

Data were obtained from the medical records of all patients with burn in AE-FUTHA managed during the study period. Information was collected on socio-demographic characteristics, clinical presentation, and management outcomes. All pregnant women who had burns were included in the study.

\section{Study area}

This study was conducted at Burns and Plastic Surgery unit of the Department of Surgery, Alex Ekwueme Federal University Teaching Hospital Abakaliki, Ebonyi State. Ebonyi State is one of the five states in the southeast geopolitical zone of Nigeria. It has a total land mass of $5,533 \mathrm{~km}^{2}$ and a population of about 3,046,28715.

Alex Ekwueme Federal University Teaching Hospital Abakaliki is a 720 bed hospital with 13 clinical departments, of which Department of Surgery is one. The Burns Unit of the Hospital is run by the plastic surgery division of surgery department. There are 8 beds in the acute burns section and 12 beds in the chronic burns and wound section. The plastic surgery division is currently run by 6 consultant plastic surgeons, 6 resident doctors, 14 specialist nurses, and 6 other nurses. Patients are admitted to the acute burns unit through the accident and emergency unit of the hospital. All the patients were co-managed with the Obstetricians and other subspecialists as indicated.

\section{Study population}

The study population was pregnant women who suffered burns injuries and were managed at AE-FUTHA during the study period. The diagnosis of pregnancy was from history, examination and confirmed by serum pregnancy test and abdominal ultrasound scan. All women who tested positive for pregnancy and had suffered burns injuries were recruited for the study irrespective of their gestational age and parity.

\section{Data analysis}

Data were obtained from the patient's medical case notes using a predesigned proforma. Data were collected on patient's age, gestational age, type, site and extent of burns injury, surgical care, complications, duration of hospital stay, and maternal and fetal outcome. Information on maternal outcome, duration of hospital stay, presence of obstetric morbidity and mortality were collected. Fetal outcome were assessed in relation to the status of the fetus at birth, early neonatal deaths, need for/and indication for newborn intensive care unit admission. Data were analyzed with IBM SPSS Statistics version 20 (IBM Corp., Armonk, NY, USA).

\section{Results}

During the study period, a total of 222 cases of burns were managed at AE-FUTHA, seventy-eight were within the reproductive age group (15 to 49 years) but only 8 were pregnant, giving an incidence of $3.6 \%$. The median age of patients was 25-34 years. The majority were government employees $(37.5 \%)$.

\section{Table 1: Socio-demographic characteristics of patients}

\begin{tabular}{ll}
\hline Variables & Frequency (\%) \\
Age(years) & $1(12.5)$ \\
$<20$ & $3(37.5)$ \\
$20-29$ & $3(37.5)$ \\
$30-39$ & $1(12.5)$ \\
$>40$ & \\
Level of education & $0(0.0)$ \\
Primary & $4(50.0)$ \\
Secondary & $4(50.0)$ \\
Tertiary & \\
Occupation & $1(12.5)$ \\
Trading & $3(37.5)$ \\
Civil servant & $2(25.0)$ \\
Public servant & $1(12.5)$ \\
Student & $1(12.5)$ \\
Farming & \\
Parity & $7(87.5)$ \\
$1-4$ & $1(12.5)$ \\
$\geq 5$ & \\
Gestational age (weeks) & $1(12.5)$ \\
$1-13$ & $5(62.5)$ \\
$14-28$ & $2(25.0)$ \\
$>28$ &
\end{tabular}


Fifty percent of the women had secondary and tertiary levels of education respectively. Similarly, fifty percent of the patients were grand-multiparous (delivered 5 or more times). Almost Two-third; $5(62.5 \%)$ had burn injury during the second trimester, while $25 \%$ occurred in the third trimester. (Table1)

Table 2: Surface area burnt, degree of burn, duration of hospital

stay and associated injuries

\begin{tabular}{lcc}
\hline Variables & Frequency & Percen \\
TBSA (\%) & 25 \\
$\leq 10$ & 2 & 25 \\
$11-20$ & 3 & 37.5 \\
$21-30$ & 1 & 12.5 \\
$31-40$ & 1 & \\
Depth of burns & & 12.5 \\
First degree & 2 & 25.0 \\
Second degree & 5 & 62.5 \\
Mixed thickness & 5 & \\
Length of hospital & stay & \\
(days) & 4 & 50.0 \\
$1-30$ & 25.0 \\
$31-60$ & 2 & 25.0 \\
$61-90$ & &
\end{tabular}

Associated injuries

Yes

2

25.0

No

6

$75.0 \%$

Table 2 showed that $37.5 \%$ of our study population had TBSA ranging between $21-30 \%$ while fifty percent of the women had TBSA of burn that was $20 \%$ and less. None of the cases under study had more than $40 \%$ TBSA. The majority $(62.5 \%)$ had mostly superficial thickness burn and complications was seen in $25.0 \%$ of the cases.

Twenty-five percent of the cases had other associated injuries. The length of hospital stay is shown in Table 2.

Table 3 shows obstetric and fetal outcomes

Figure 1, shows the etiology of burns in pregnancy. Flame injury was the commonest $(62.5 \%)$ cause of burn followed by scald injury $(25.0 \%)$ and friction $(12.5 \%)$ related injury which was seen among the women who had road traffic accident.
Table 3: Clinical findings of the Burns suffered by the study population

\begin{tabular}{lll}
\hline TBSA (\%) & Frequency & Percentage \\
$\leq 10$ & 2 & 25 \\
$11-20$ & 2 & 25 \\
$21-30$ & 3 & 37.5 \\
$31-40$ & 1 & 12.5 \\
Depth of burns & & \\
First degree & 1 & 12.5 \\
Second degree & 2 & 25.0 \\
Mixed thickness & 5 & 62.5 \\
Length of hospital stay (days) & & \\
1-30 & 4 & 50.0 \\
$31-60$ & 2 & 25.0 \\
$61-90$ & 2 & 25.0 \\
Associated injuries & & \\
Yes & 2 & 25.0 \\
No & 6 & 75.0 \\
\hline
\end{tabular}

TBSA- total body surface a

Table 4: Obstetric and Fetal outcome

\begin{tabular}{lll}
\hline Variable & Frequency & Percentage \\
Timing of delivery & 7 & 87.5 \\
Term & 1 & 12.5 \\
Preterm & & \\
Mode of delivery & 7 & 87.5 \\
Spontaneous vaginal delivery & 7 & 12.5 \\
Caesarean section & 1 & \\
Maternal mortality & & 100.0 \\
None & 8 & \\
Fetal outcome & & 100 \\
Live birth & 8 & 12.5 \\
Early neonatal death & 1 & \\
Birth weight (kg) & & 25.0 \\
$<2.5$ & 2 & 37.5 \\
$2.5-3.5$ & 3 & 37.5 \\
$>3.5$ & 3 &
\end{tabular}

Most (62.5\%) of the burn injuries occurred during the dry and Harmattan seasons (between October and March), while $37.5 \%$ occurred between April and September- Figure 2. Fifty percent of the burns occur in open kitchen, $12.5 \%$ in enclosed kitchen; making a total of $62.5 \%$ of accident occurring the kitchen, $25 \%$ happened in the living room and $12.5 \%$ occurred on the road. Figure 3. All the burn injuries were accidental, no suicidal attempt and no case of assault were recorded.

There were no inhalational injuries and $75 \%$ of the patients had no other associated injuries but $25 \%$ sustained some other forms of injuries such as pubic symphysis diastasis $(12.5 \%)$ and avulsion injury of the right heel (12.5\%). 


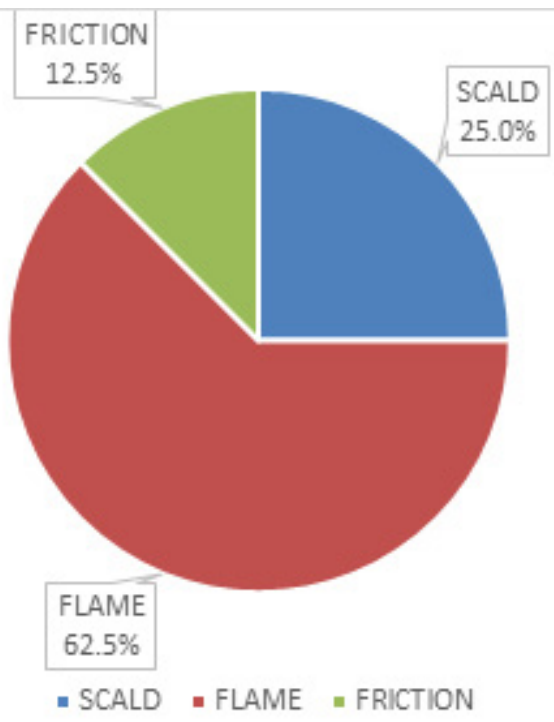

and burns involving the trunk, while open dressing was used for the facial burns which constituted $37.5 \%$ of cases. All the patients were co-managed with Obstetricians, $25 \%$ with the Physiotherapist, $12.5 \%$ with the orthopedic surgeons and $25 \%$ with the Physicians (Cardiologist).

The majority $(87.5 \%)$ of the women delivered their babies at term, while $12.5 \%$ had premature delivery at 28 week. Also, about $87.5 \%$ were delivered via spontaneous vaginal delivery, while $12.5 \%$ was delivered through pre-burn injury planned elective caesarean section (Table 3). Over a-third (37.5\%) had wound infection due to Pseudomonas aeruginosa. There was no maternal death; however the only case delivered prematurely at 28 weeks had early neonatal death in the neonatal intensive care unit (NICU) on the third day of life. Half $(50 \%)$ of the patients were discharged within the first month of admission, $25 \%$ had hospital stay of 2 months and 3 months respectively.

\section{Figure 1: causative agents/source of injury}

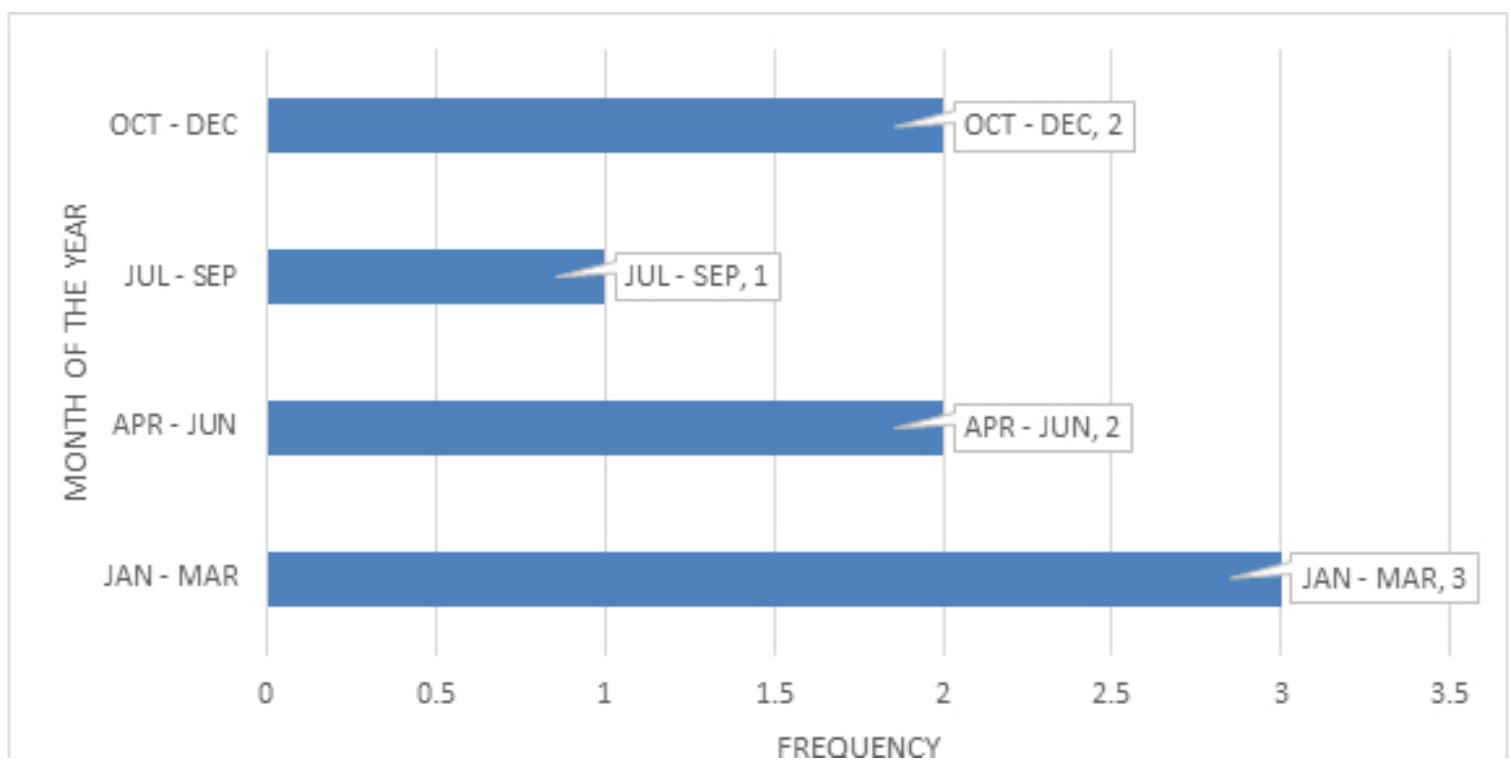

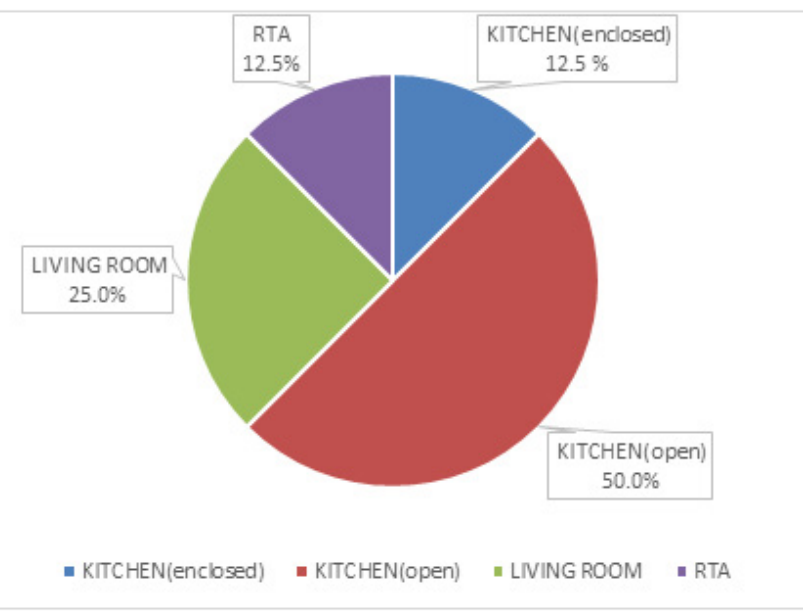

Figure 3: Location of burn occurrence

All the patients had various degrees of both upper and lower extremities burn; $50 \%$ had anterior trunk, $37.5 \%$ facial and $12.5 \%$ breast involvements.

Fifty percent $(50 \%)$ of the patients had split-thickness skin graft, while the remaining were managed with conventional wound dressings and adjunctive wound therapies. Occlusive dressing were used for all the patients with extremity burns

\section{Discussion}

Pregnancy increases the challenges in the management of burns patients because it demands special management and requires early and adequate resuscitation and the use of antimicrobial agents whose use may be influenced by safety concerns. The present study showed that the incidence of burns in pregnancy was $3.6 \%$ and that most incidents occurred after the first trimester. Multi-disciplinary approach to management was associated with good surgical and fetomaternal outcomes for those who delivered at term.

The reported incidence of burn in pregnancy varies from region to region. The incidence of $3.6 \%$ found in this study is lower than those reported by Ogbogu et al. $(9.11 \%)^{5}$, Zulquarnain et al. $(7.2 \%)^{16}$ and Jain et al. $(13.3 \%)^{17}$. The true incidence of burns in pregnancy may be underestimated as pregnancy test is not routinely performed on all women of reproductive age group who present with burns.

There are reports indicating that flame burns from gas and kerosene which are commonly used as fuel for cooking and powering machines are the most common causes of burns in most low and middle income countries including Nigeria ${ }^{4,9,18-20}$. This finding is similar to that of the current study where majority of burn in pregnancy were caused by flame and occurred in open kitchens. Adulteration of petroleum product is thought to be responsible for explosion 
and flame burns, especially during seasonal scarcity of petroleum products in Nigeria ${ }^{21}$.

This study also showed that burns injury in pregnancy was commoner between October and March (62.5\%) of each year, this corresponds to dry season and harmattan period (dry and windy period). This finding is consistent with the report by Isiguzo et al. in Imo state, Nigeria in their two year prospective study on burn injuries ${ }^{22}$. The reason is partly because the dryness and winds of harmattan season aids combustion. Also the cold nights and early morning weather during the harmattan period increases the desire for warmth with lighting of stoves, firewood and boiling of water for bathing. These increase the risk of getting burnt. However, contrary to our finding, Momoh et al reported that more burns injuries occurred during the rainy season ${ }^{23}$.

There was no maternal death recorded in the current study; however perinatal mortality was recorded in a patient who was delivered an extreme preterm baby at 28 weeks gestation. Conversely, some studies have reported maternal mortality figures between $28.3 \%$ and $63 \% 4,5,8,19$. Factors that have been associated with poor maternal and perinatal outcomes include TBSA $>50 \%$, second and third-degree burns, inhalational injuries, hypovolemia, septicemia, comorbidities, and suicidal tendencies. None of the patients in this study had more than $40 \%$ TBSA. There were also no inhalational injuries. Adequate fluid and antibiotics were also provided to maintain hemodynamic circulation, prevent electrolyte derangement and infection.

The good maternal outcome recorded in the present study may have been due to early presentation, timely and adequate resuscitation and management of patients in high dependency unit (acute burn ward). Multi-disciplinary management with the obstetrician and other specialists was also an important contributor. Some authorities have recommended that viable pregnancies $>32$ weeks should be delivered early after resuscitation, if the TBSA is above $40 \%$, to enhance fetal survival and that pregnancy should be terminated irrespective of the gestational age, if the TBSA is above $50 \%{ }^{15}$.

\section{Conclusion}

Most burn injuries in pregnancy were seasonal and occurred after the first trimester with majority of pregnancies carried to term. Surgical and obstetric outcomes were favorable with optimal fetal and maternal outcomes and no attendant increase in operative delivery in cases which occurred during the third trimester. Early involvement of obstetricians in all burns affecting pregnant women is advised especially in burn centres where obstetricians are hardly in the employ.

\section{Strength and Limitation}

The strength of this study was mainly the availability of complete records. Additionally, the data in the present study were quite recent, reflecting current trends. However, its limitations are related to the small sample size, which may have been affected by missed cases of early pregnancy. There is need to rule out pregnancy in all females burns patients within reproductive age group who are sexually active. There is also need for larger prospective multi-centre studies, meta-analysis or systematic reviews to help provide high level evidence needed to develop and update protocols in management of pregnant burns patients.

\section{Author Contributions}

Authors' contributions Nnadozie UU: participated in the conceptualisation, study design, data collection/analysis and interpretation of finding and drafting of the manuscript. Okorie GM and Maduba CC : participated in study design, interpretation of findings and drafting of the manuscript. Anikwe CC and Ugbala A: participated in the data analysis and the interpretation of finding, Lawani OL and Asiegbu OGK: participated in the interpretation of findings and drafting of the manuscript. All participated in the review of the final manuscript. All the authors approved the manuscript

\section{Acknowledgements: None}

Competing interests: The authors declare that no competing interests exist.

\section{Funding}

This researchers did not receive any specific grant from funding agencies in the public, commercial, or not-for-profit sectors for this study.

\section{References}

1.Ravi C. History, Epidemiology, and Prevention of Burns. In:Sujata S, Tiwan VK and Arun G (eds): Principles and Practice of Burn Care. St Loius USA. Jaypee Brothers. 2010. p 3 - 17.

2.Njoku OI, Joannes UO, Christian MC, Azubike OK. Trauma during pregnancy in a Nigerian setting: Patterns of presentation and pregnancy outcome. Int J Crit Illn Inj Sci. 2013;3(4):269-273. doi:10.4103/22295151.124155

3.Lawani LO, Iyoke CA, Onoh RC et al. Obstetric benefits of health insurance: a comparative analysis of obstetric indices and outcome of enrollees and non-enrollees in southeast Nigeria. J Obstet Gynaecol 2016 Oct; 36 (7): 946-949.

4.Rezavand $\mathrm{N}$ and Seyedzadeh A. Maternal and fetal outcome of burns during pregnancy in Kermanshah, Iran. Ann Burns Fire Disasters. 2006 Dec 31; 19(4):174-176.

5.Ogbogu CJ, Uduezue AO, Ikpemhi AW, Agunwa C. Burns injuries in pregnancy in a regional burn center in Nigeria: Presentation, maternal and fetal outcome. Burns Open. 2018 Jan, 2(1):53-58.

6.Subrahmanyam M. Burns during pregnancy- Effect on maternal and fetal outcomes. Ann Burns Fire Disasters. 2006 Dec 31; 19(4):177-179.

7.Chandra G, Gaurav K, Kumar S et al. Burns during pregnancy: A retrospective analysis of 19 cases. Arch Intl Surg. 2016; 6(1):28-31.

8.Mabogunje OA. Burns injuries during pregnancy: an African series. J Natl Med Assoc 1990 Sep. 82(9): 641 - 644.

9.Agarwal P. Thermal injury in pregnancy. Predicting maternal and fetal outcome. Indian J. Plast Surg. 2005; 38(2):95-99

10.Guo SS, Greenspoon JS, Kahn AM. Management of burns injuries during pregnancy. Burns 2001; 27:394-7

11.Bartle EJ, Sun JH, Wang XW. Burns in pregnancy. J. Burn Care Rehabil 1988; 9:485-7

12.DeitchEA, Rightmire DA, Clithier J, Blass N. Management of Burns in Pregnant women. Surg. Gynecol Obstet. 1985 Jul, 161(1): 1-4

13.Napoli B, D’ Arpa N, Masellis M, Graziano R. Burns in pregnancy. Ann Burns Fire Disasters. Vol XIII-no 1- March 2000.Available at: http://www.medbc.com/annals/review/vol_13/num_1/text/vol13n1p18. htm. Accessed 14 July 2019.

14.Nnadozie UU, Umeokonkwo CD, Maduba CC, Igwe-Okomiso D, Onah CK, Madubueze UC et al. Antibiotic us e among surgical inpatients at a tertiary health facility: a case for a standardized protocol for presumptive antimicrobial therapy in developing world. 
Infection Prevention In Practice, 2020;2(4): https://doi.org/10.1016/j. infpip.2020.100078.

15.Ebonyi State- Nigeria Investment Promotion Commission. https:// nipc.gov.ng. Accessed 28th August 2020

16.Zulquarnain M, Imran A, Fahad K, Ansarul H. Pregnancy in burns: maternal and fetal outcome. Indian J burns. 2012; 20(1): 36-41. Jain ML, Garg AK. Burns with pregnancy- a review of 25 cases. Burns, 1993; 19: 166-167.

17.Mehdi P, Ali AM, Abdolreza R, Masoud N, HainidRk, Davood M. Epidemiology of burns during pregnancy in southern Iran: effect on maternal and fetal outcome. World Appl Sci J, 2013; 28(2): 153-158.

18.Akhtar, MA, Mulawkar PW, Kulkarni HR. Burns in pregnancy: effect on maternal and fetal outcome. Burns, 1994; 20(4): 351-355.
19.Chama C, Na'Aya HU: Severe burns in pregnancy in Northern Nigeria. J Obstet Gynaecol, 2002, 22: 20-22.

20.Dongo AE, Eshobo EI. A 5-year review of burn injuries in Irrua. BMC Health Serv Res. 2007, 7:171

21.C.M. Isiguzo, C. Opara, U.U. Nnadozie, K. Opara, Burn injury in tertiary health facility in South East Nigeria: A 2 year prospective study, Burns Open (2020), doi: https://doi.org/10.1016/j.burnso.2020.08.001

22.Momoh MI, Okugbo SU, Ohanaka EC, Ugbeye ML. Causes and Risk factors in burns. Experience from Benin City. Momoh MI, Okugbo SU, Ohanaka EC, Ugbeye ML. Causes and Risk factors in burns. Experience from Benin City. Niger J Surg Sci 2000; 10: 103-111. 